\title{
Tinea Capitis in a Healthy Adult: An Unexpected Diagnosis Made on Dermoscopy
}

\author{
Enzo Errichetti ${ }^{1}$, Cinzia Buligan ${ }^{1}$
}

1 Dermatology Institute, “Santa Maria della Misericordia” University Hospital, Udine, Italy Citation: Errichetti E, Buligan C. Tinea capitis in a healthy adult: an unexpected diagnosis made on dermoscopy.
Dermatol Pract Concept. 2021; 11(4):e2021083. DOI: https://doi.org/10.5826/dpc.1104a83

Accepted: February 22, 2021; Published: October, 2021

Copyright: $\odot 2021$ Errichetti et al. This is an open-access article distributed under the terms of the Creative Commons Attribution License BY-NC-4.0, which permits unrestricted noncommercial use, distribution, and reproduction in any medium, provided the original authors and source are credited.

Funding: None.

Competing interests: None.

Authorship: Both authors have contributed significantly to this publication.

Corresponding author: Enzo Errichetti, MD, Dermatology Institute, “Santa Maria della Misericordia” University Hospital. Piazzale Santa Maria della Misericordia, 15.33100-Udine, Italy. Email: enzoerri@yahoo.it

\section{Case Presentation}

A 37-year-old healthy male presented with a 2-month history of itchy, scaly, erythematous patches on the scalp, displaying broken hairs in some areas (Figure 1, A and B). Medical history (including immune system/infectious diseases) was unremarkable, and no medication was being taken. At a first glance, the main clinical suspect was scalp psoriasis with broken hairs resulting from continuous scratching. Besides broken hairs, erythema and white scaling, dermoscopic examination revealed findings contrasting with the clinical suspect, ie comma hair, zig-zag hairs, corkscrew hair and Morse code hair (Figure 1, C and D). A fungal culture from lesional scales was performed and Microsporum Canis was isolated, consistently with the diagnosis of tinea capitis. An
8 -week course of oral Terbinafine $250 \mathrm{mg} /$ die was prescribed with complete healing.

\section{Teaching Point}

Tinea capitis is typically considered a disease of childhood or immunosuppressed patients, and postmenopausal women, yet healthy adults may also develop it [1]. The rarity of the condition in this category of patients is often responsible for diagnostic delays or mistreatments [1]. In this regard, dermoscopy may increase the index of suspicion by showing specific features resulting from fungal invasion of the hair shaft which may cause a structural weakness of the infected hairs, with consequent more or less marked bends (viz. comma, corkscrew, or zig-zag hair) or irregularly narrowed pale areas (Morse code hair) [2]. 


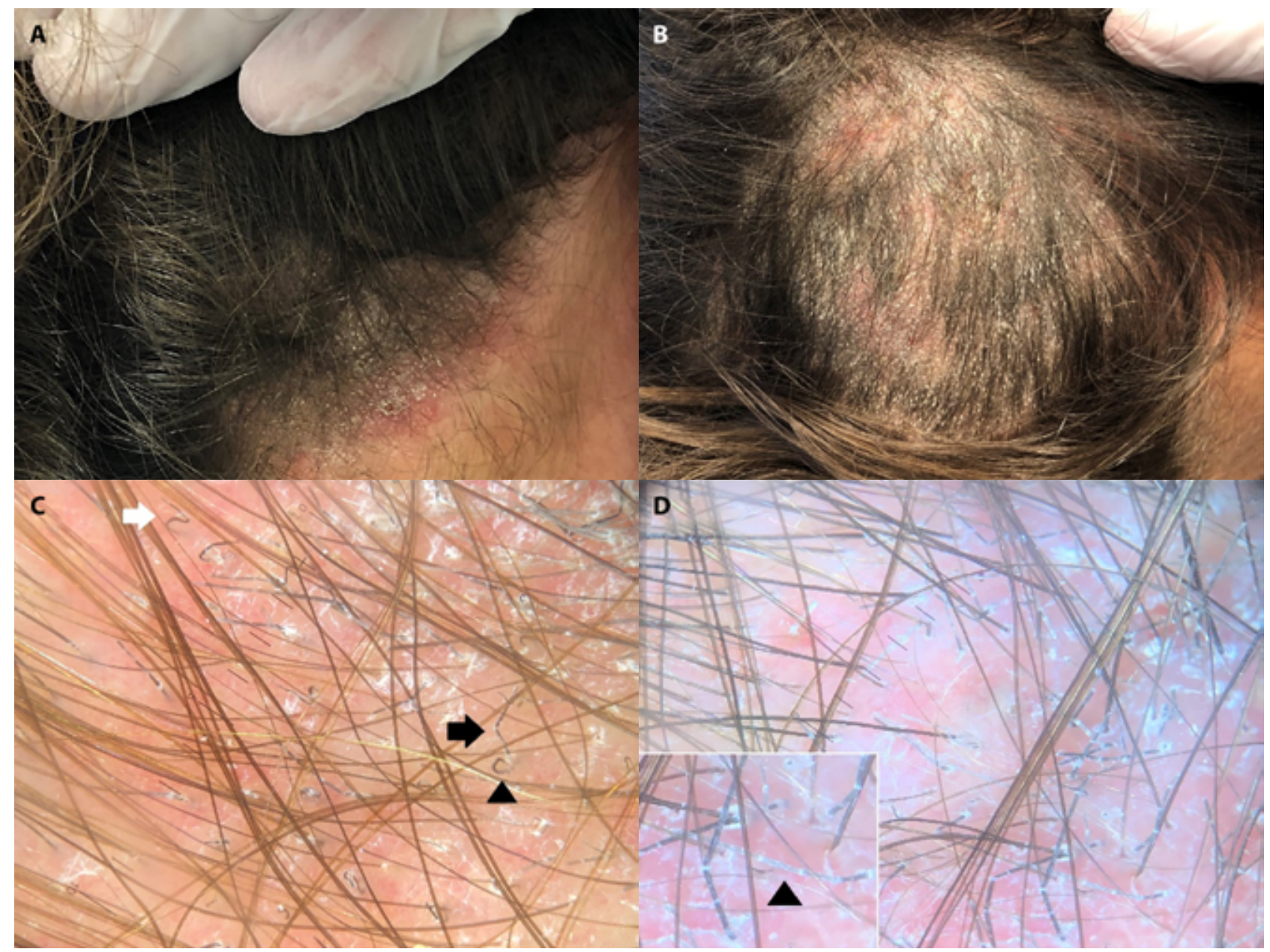

Figure 1. (A, B) Clinical examination shows erytheamatous-desquamative patches of the scalp. (B) Broken hairs are also visible. (C) Dermoscopy (Polarized light; x10 magnification) reveals unspecific erythema and white scales along with comma hair (black arrowhead), corkscrew hair (white arrow) and zigzag hair (black arrow). (D) Morse code hair (hair shafts presenting alternating whitish and brown bands) (black arrowhead in the inset) are also present.

\section{References}

1. Buckley DA, Fuller LC, Higgins EM, du Vivier AW. Lesson of the week. Tinea capitis in adults. BMJ 2000; 320: 1389-90. DOI: 10.1136/bmj.320.7246.1389. PMID: 10818032. PMCID: PMC1118052.
2. Waśkiel-Burnat A, Rakowska A, Sikora M, Ciechanowicz P, Olszewska M, Rudnicka L. Trichoscopy of Tinea Capitis: A Systematic Review. Dermatol Ther (Heidelb) 2020; 10: 43-52. DOI: 10.1007/s13555-019-00350-1. PMID: 31907867. PMCID: PMC6994564. 